\title{
Logic Formulation and Evaluation of Academic Constraints
}

\author{
Aftab Ahmed ${ }^{1}$, Walayat Hussain ${ }^{2}$, Ali Kamran ${ }^{3}$ \\ ${ }^{1,2}$ Department of Computer Science, BUITEMS, Quetta \\ Email: aftab.ahmed@buitms.edu.pk \\ Email: walayat.hussain@ buitms.edu.pk \\ ${ }^{3}$ Institute of Space Technology, Islamabad \\ Email: Ali.kamran@ist.edu.pk
}

\begin{abstract}
University curriculum scheduling is a prominent research issue of resources optimization. The problem consists of constraints, composite event variables and their placement domains. This research work introduces a novel set of evaluation heuristics those sharply scan out the dataset with respect to hard or soft constraints and consequently assign penalties to conflicting events. The research approach is examined over a number of diverse benchmark dataset where complexity increases with subject to their number of events, constraints and curriculums. Furthermore, each dataset is classified over six complexity scales where each scale is differentiated set of constraints. The prime advantages are revealed from the research work to acquire accurate status of constraints violations with respect to various datasets and complexity scales which leads to obtain optimal solution in short span of time and using less computational resources.
\end{abstract}

Keywords: Evaluation Function, Hard Constraints, Benchmark datasets, Penalty function

\section{Introduction}

Academic Scheduling is an important combinatorial optimization problem. The problem can be defined by assignment of instructors, courses and room under various types of constraints. The key objective is to minimize number of conflicts and maximize resources utilization. The problem is widely considered as challenging resources assignment job since interdependent constraints take into account. The classic scheduling problem precisely illustrated by constraints, composite variables (events), finite domain of values and violation penalty costs. 
The typical solution consists to assign the suitable site (venue or room), personnel resource (teacher) on defined time length (working day and timeslot) for the enrolled group of students (Curricula). The framework schema is separated under two types, the hard and the soft constraints. In practice, the hard constraints are mandatorily required to be satisfied under all circumstances. In fact, a solution qualifies essential feasible state if only, all the hard constraints conflicts get resolved. Nearly all the hard constraints are commonly adapted and exercised in academic institutes; nevertheless several types of soft constraints can be at variance from each other with subject to their own educational priorities. The research work presented in this article causes the successful outcome of relevant work (Aftab Ahmed et al., 2011a)

- Time Elements (Days, Periods and timeslots): A five to six number of teaching/working days are usually exercised. Each day is divided into predetermined duration of various sessions in some cases in range of three to six. A timeslot is single entry of lecture event. A period contains a stack of timeslots, maximum number of rooms can provide larger stack of timeslots.

- Courses and curriculum: A course is offered to group of students which may be separated in multiple sessions throughout the week. On the other hand a curriculum is correlated set of courses which usually comprises over rudiments (prerequisite), optional (elective) and mandatory (core) courses. Student(s) can get enrollment in a range of courses to be bound with curricula.

- Physical resources (Class Room, Lab and Multimedia Projector etc.): the necessity of technical equipment like projector, smart board etc or required venue like auditorium or seminar hall etc which is highly desirable to particular event.

Next section takes a look on state-of-the-art development in the field of scheduling. In section Three, typical curriculum problem has been illustrated categorically. At the first, timetabling terminology is described. The most important components of timetabling, the hard and soft constraints are sorted out which are handled globally in most of the problem instances. Afterward, benchmark instances are described with distinguished classifications which contain five conventional and one additional complexity scale. Constraints are exemplified through figures and mathematical equations.

\section{Related Work}

The classic university scheduling is described as (M.W. Carter and Laporte, 1998): "a multi-dimensional assignment problem in which students, teachers (or faculty members) are assigned to courses, course sections or classes; events (individual meetings between students and teachers) are assigned to classrooms and times" In combinatorial optimization problem such as curriculum scheduling, 
a fitness or heuristic function keeps immense importance in general. An evaluation function (M. Adriaen et al., 2003) is scale of constraints violations and their relevant cost parameters. Ghaemi (Ghaemi et al., 2007) draw an evaluation function that examine an array of constraints subject to constant plenty. Nevertheless, sometimes evaluation function is supposed to produce bit more quality performance when it is used to exploit the resources usage. In addition, ideally an evaluation function is designed purposely to acquire non violated outcome, which is someway not likely in huge and complex real world or benchmarks datasets and eventually it has to compromise over small number of unsolved soft violations.

Every scheduling issue can be recognized by Constraint Satisfaction Problem (CSP) that comprises over three key elements finite set of variables, their domains and hard \&soft constraints (V, D, and C). The merits (W. Legierski, 2002) of CLP is stated "a straightforward statement of the constraints serves as part of the program. This makes the program easy to modify, which is crucial in real-world problems" in addition, (G. M. White and J. Zhang, 1998) "when problems are formulated as constraint logic problems they become known as Constraint Satisfaction Problems, (CSP)".

Aftab and Li (Aftab Ahmed and Li, 2010) successfully solved a read world case study using Constraint Satisfaction Problem in two separate phases where first one completely sweep out hard constraints and later the second phase deliberately deals only with the soft constraints. Their extended work in (Aftab Ahmed et al., $2011 b$ ) appended another phase to acquire very optimal outcome by implementation of Genetic Algorithm over partial solution.

\section{Problem Explanation}

The combinatorial problem consists of numerous variables and constraints including Teacher, class rooms, courses, time-slots and group of student. Precisely Scheduling Problem $=[\mathrm{I}, \mathrm{T}, \mathrm{C}, \mathrm{S}, \mathrm{G}]$. Curriculum schema is categorized under two major types, hard and soft constraints. The set of hard constraints (HC) is decisively required not to be satisfied. In contrary, all the soft constraints cannot be removed from the benchmark as well as real world dataset so highest degree of soft satisfaction places the solution on higher order of quality scale.

\subsection{Constraints and Dynamic Penalty Scale}

As it is discussed, Hard Constraints should be resolved under all circumstances. Generally, an inclusive set of constraints is immersed in most of the academic institutes; though a certain number of adapted constraints are also fabricated. The possibility of violation likely rises while two or more events need a shared resource at the same time.

Penalty formulation comprises over several algorithms, each algorithm scan out the dataset for particular constraint and if conflict finds then stamp that event 
penalty cost. The cost mounts with affiliated conflict with that event. The penalty schema is precisely illustrated in 3.2.2 Classroom Conflicts (HC2): No more than one lecture evens should be given to a teacher, a hard violation is counted in such case. Conflicted pair of events is assigned by equal penalty but every time penalty multiplies with repeated occurrence of the violation. E.g. Layout.Slot $=$ HC. Penalty $\times \sum_{k=1}^{n} E \boldsymbol{v t}$

$$
\sum_{\mathrm{i}=1}^{\text {Peroid }} \sum_{\mathrm{j}=1}^{\text {Faculty }} \mathrm{f}_{\mathrm{j}} \cdot \mathrm{c}_{\mathrm{i}} \leq 1
$$

Table 1 that dynamically grow complex on the basis of each scale. This penalty schema (conflicting cost of hard and soft constraints violations) is advised by Bonutti (Alex Bonutti et al., 2010) however in this research paper, one advanced complexity scale is appended for all benchmark datasets to examine solving capability of research approach. Each complexity scale brings up the diverse penalty weights and intensity in for identical constraints. Moreover, number of constraints and lecture events increases in with respect to available resources. These discrepancies stand each dataset different from other so consequently it requires generic as well as robust evaluation and solving approaches. The constraints which are recognized by variables HC (Hard Constraint) in 3.2.2 Classroom Conflicts (HC2): No more than one lecture evens should be given to a teacher, a hard violation is counted in such case. Conflicted pair of events is assigned by equal penalty but every time penalty multiplies with repeated occurrence of the violation. E.g. Layout.Slot $=$ HC. Penalty $\times \sum_{k=1}^{n} \boldsymbol{E} \boldsymbol{v} \boldsymbol{t}$

$$
\sum_{\mathrm{i}=1}^{\text {Peroid }} \sum_{\mathrm{j}=1}^{\text {Faculty }} \mathrm{f}_{\mathrm{j}} \cdot \mathrm{c}_{\mathrm{i}} \leq 1
$$

Table 1 are mandatory for the state of feasible solution whereas rests of the group is known as soft constraints.

\begin{tabular}{|c|c|c|c|c|c|c|}
\hline & $\begin{array}{l}\text { Lectures } \\
\text { Cearrence }\end{array}$ & & Classromen & {$\left[\begin{array}{c}\text { Reom } \\
\text { Oceupaney }\end{array}\right.$} & E & E \\
\hline Day & Room No & Session - 1 & Session - 2 & Session - 3 & Sessio & $n-4$ \\
\hline \multirow{7}{*}{$\begin{array}{l}\frac{\pi}{\partial} \\
\frac{0}{2} \\
\frac{0}{2}\end{array}$} & Room 1 & Cur1, s13, T02 & Cur1, S14, T07 & Cur1, S15, T14 & & \\
\hline & Room 2 & Cur1, S23, T04 & Cur2, S25, T11 & Cur3, S35, T13 & Cur3, S3 & 1, T15 \\
\hline & Room 3 & Cur4, S42, T07 & Cur9, s94, T12 & Cur4, S44, T11 & Cur4, S4 & I, T06 \\
\hline & Room 4 & Cur5, S53, T05 & Cur5, S55, T12 & Cur5, S51, T05 & & \\
\hline & Room 5 & Cur6, S65, T13 & Cur8, S83, T02 & Cur6, S61, T15 & & \\
\hline & \multirow{2}{*}{ Room 6} & Cur7, s74, T08 & Cur7, s73, T10 & Cur7, s71, T12 & & \\
\hline & & Cur8, S81, T14 & Cur6, S62, T14 & Cur8, S84, T08 & Cur8, S8 & 5, T03 \\
\hline
\end{tabular}

\subsection{Hard Constraints Description}

Fig: exclusively depicts the different types of hard constraints, multiple assignments of curriculum (Curl under $\mathrm{HC}_{1}$ tag) at the same time and session causes violation.

Similarly replication of resource person highlighted with tag $\mathrm{HC}_{3}$ in room 3 and 4 respectively. The violation $\mathrm{HC}_{3}$ is taken place due to not inadequate seating 
availability in room number 6 ; on the other hand $\mathrm{HC}_{4}$ is happened because of prior notified unavailability of teacher $\mathrm{T}^{2} 6 . \mathrm{HC}_{5}$ is special type of hard or soft constraint depends on the complexity scale and institutional need. As it is observed in session four, manifold events are assigned in the same venue and time cause $\mathrm{HC}_{5}$ to take place.

3.2.1 Lectures recurrence $\left(\mathbf{H C}_{\mathbf{1}}\right)$ : Multiple events should not be assigned to a group of students concurrently.

$$
\sum_{\mathrm{i}=1}^{\text {Peroid }} \sum_{\mathrm{j}=1}^{\text {Courses }} \mathrm{g}_{\mathrm{i}} \mathrm{c}_{\mathrm{j}} \leq 1
$$

3.2.2 Classroom Conflicts (HC2): No more than one lecture evens should be given to a teacher, a hard violation is counted in such case. Conflicted pair of events is assigned by equal penalty but every time penalty multiplies with repeated occurrence of the violation. E.g. Layout.Slot $=$ HC. Penalty $\times \sum_{k=1}^{n} \boldsymbol{E} \boldsymbol{v t}$

$$
\sum_{\mathrm{i}=1}^{\text {Peroid }} \sum_{\mathrm{j}=1}^{\text {Faculty }} \mathrm{f}_{\mathrm{j}} \cdot \mathrm{c}_{\mathrm{i}} \leq 1
$$

\begin{tabular}{|c|c|c|c|c|c|c|c|c|}
\hline Var. & Type & Constraint Title & Scale $_{1}$ & Scale $_{2}$ & $\mathrm{Scale}_{3}$ & $\mathrm{Scale}_{4}$ & Scale $_{5}$ & Scale $_{6}$ \\
\hline $\mathrm{HC}_{1}$ & \multirow{5}{*}{ 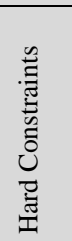 } & Lectures recurrence & $\infty$ & $\infty$ & $\infty$ & $\infty$ & $\infty$ & $\infty$ \\
\hline $\mathrm{HC}_{2}$ & & Room Conflicts & $\infty$ & $\infty$ & $\infty$ & $\infty$ & $\infty$ & $\infty$ \\
\hline $\mathrm{HC}_{3}$ & & Room Occupancy & $\infty$ & $\infty$ & $\infty$ & $\infty$ & $\infty$ & $\infty$ \\
\hline $\mathrm{HC}_{4}$ & & Availability & $\infty$ & $\infty$ & $\infty$ & $\infty$ & $\infty$ & $\infty$ \\
\hline $\mathrm{HC}_{5}$ & & Room Suitability & - & - & 3 & $\infty$ & - & - \\
\hline $\mathrm{SC}_{1}$ & \multirow{9}{*}{ 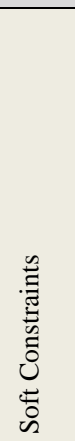 } & Room Capacity & 1 & 1 & 1 & 1 & 1 & - \\
\hline $\mathrm{SC}_{2}$ & & Min Working Days & 5 & 5 & - & 1 & 5 & 5 \\
\hline $\mathrm{SC}_{3}$ & & Isolated Lectures & 1 & 2 & - & - & 1 & 2 \\
\hline $\mathrm{SC}_{4}$ & & Windows & - & - & 4 & 1 & 2 & 1 \\
\hline $\mathrm{SC}_{5}$ & & Room Stability & - & 1 & - & - & - & 2 \\
\hline $\mathrm{SC}_{6}$ & & Student Min Max Load & - & - & 2 & 1 & 2 & 1 \\
\hline $\mathrm{SC}_{7}$ & & Travel Distance & - & - & - & - & 2 & - \\
\hline $\mathrm{SC}_{8}$ & & Double Lectures & - & - & - & 1 & - & - \\
\hline $\mathrm{SC}_{9}$ & & Teaching Max Load & - & - & - & - & - & 5 \\
\hline
\end{tabular}

Table 1 Problem classification description

3.2.3 Room Occupancy (HC3): Two concurrent lecture events cannot be assigned in a single venue; each such conflict multiplies the penalty cost.

$$
\sum_{\mathrm{i}=1}^{\text {rooms }} \sum_{j=1}^{\text {Evnts }} r_{i} \cdot e_{j} \leq 1
$$

3.3.3 Room Suitability (HC4): The Class room must sufficiently be equipped with necessary hardware for example multimedia projector, white/black board etc.

$$
\sum_{\mathrm{i}=1}^{\text {rooms }} \sum_{\mathrm{j}=1}^{\text {goups }} \mathrm{r}_{\mathrm{ik}} \cdot \mathrm{g}_{\mathrm{j}} \leq 1 \text { where } \forall \mathrm{k} \in\{1 \cdots \text { Room. Equipments }\}
$$

3.3.4 Availabilty (HC5): Event should not be assigned if instructor has declared unavailability on a certain specific time because of any valid reason. 


$$
\sum_{\mathrm{i}=1}^{\text {Faculty }} \sum_{\mathrm{j}=1}^{\text {Events }} \mathrm{f}_{\mathrm{i}} \cdot \mathrm{e}_{\mathrm{j}}<>\text { True }
$$

\subsection{Soft Constraints Description}

In most of the cases almost all the hard constraints are widely available in universities however the quality scale largely depends upon the violation presence of soft constraints.

\subsection{Complexity Scale 1}

This scale comprises over four hard and three soft constraints; penalty cost Algorithm/criterion is defined bellow.

3.4.1 SC 1 (Room Capacity): Number of students exceeding the class-room seating capacity would be counted a violation.

$$
\sum_{\mathrm{i}=1}^{\text {rooms }} \sum_{\mathrm{j}=1}^{\text {goups }} \mathrm{r}_{\mathrm{ik}} \cdot \mathrm{g}_{\mathrm{j}} \leq 1 \text { where } \forall \mathrm{k} \in\{1 \cdots \text { Room. Capacity }\}
$$

3.4.2 $\mathrm{SC}_{2}$ (Min Working Days): Courses should be ordered and separated with predetermined number of sessions over working days.

$$
\sum_{\mathrm{i}=1}^{\text {Days }} \sum_{\mathrm{j}=1}^{\text {Cours }} \mathrm{d}_{\mathrm{i}} \mathrm{c}_{\mathrm{j}} \geq \mathrm{s}_{\mathrm{i}} \text { where } \forall \mathrm{k} \in\{1 \cdots \text { Course. Sessions }\}
$$

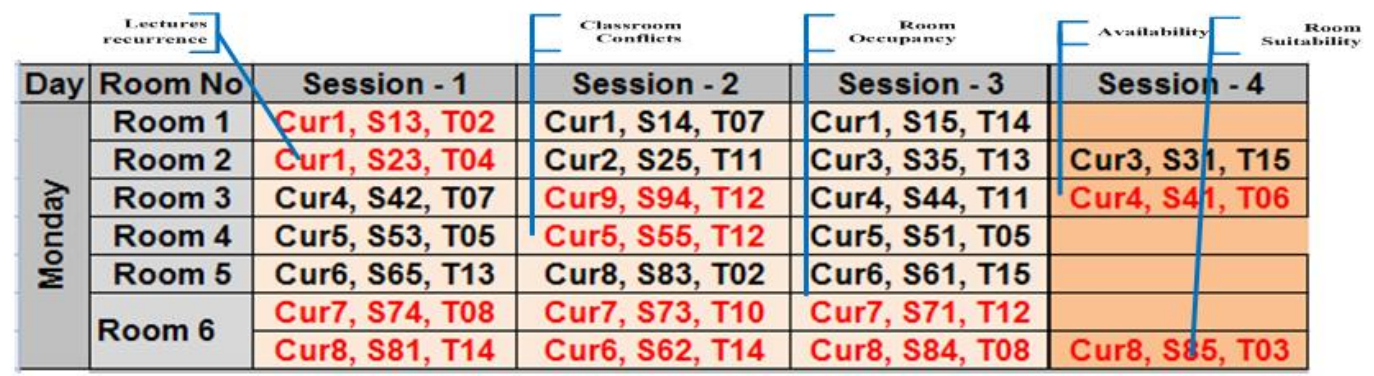

Fig. 2: Hard Constraints Demonstration

Procedure: Room Capacity

Def Min_Working_Days:

1. IF Event.Students < Room.Capacity:

2. Layout[Penalty_Slot $]=($ Room.Capacity - Event.Students $) \times$ Scale.Penalty

3. EndIf 


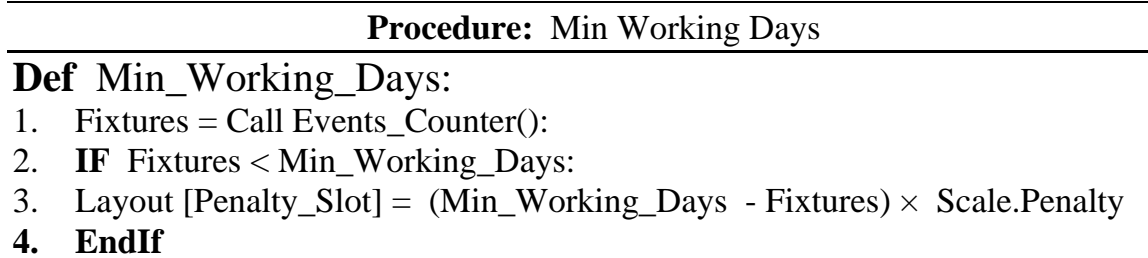

Procedure: Isolated Lectures

1. Def Isolated_Events:

2. For All Day Events:

3. IF CountDay (Event) $==1$ :

4. Layout[Penalty_Slot $]=$ Scale.Penalty

5. EndIf

6 Fnd While

3.4.3 $\mathrm{SC}_{3}$ (Isolated Lectures): Generally contiguous lecture events are appreciated by student, the window between events causes a soft violation and penalty adds up with each identical occurrence.

$$
\sum_{\mathrm{i}=1}^{\text {Days }} \sum_{\mathrm{j}=1}^{\text {Cours }} \mathrm{d}_{\mathrm{i}} \mathrm{c}_{\mathrm{j}} \leq 1 \text { where } \forall \mathrm{k} \in\{1 \cdots \text { Course. Sessions }\}
$$

\subsection{Complexity Scale 2}

This level is extended with one new constraint.

3.5.1 $\mathrm{SC}_{5}$ (Room Stability): Succeeding event(s) of each student group should be placed on the same room/venue. Number of different rooms in sequence events multiply penalty. e.g.

$$
\sum_{\mathrm{i}=1}^{\text {groups }} \sum_{\mathrm{j}=1}^{\text {events }} \beta_{\mathrm{ik}}=\text { Room }
$$

Procedure: Room Stability

1. Def Room_Stability:

2. For All Day Events:

3. IF Sequence_Placments(Event $)==$ NOT Stable:

4. Layout[Penalty_Slot $]=\sum_{1}^{\mathrm{n}}$ Room. Violations $\times$ Scale.Penalty

5. EndIf

6. End_LOOP

\subsection{Complexity Scale 3}

The scale is extended by three new soft constraints defined as bellow.

3.6.1 $\mathrm{SC}_{4}$ (Windows): The empty bubbles (sessions) between two relevant events of one group are considered as soft violations.

$$
\sum_{\mathrm{i}=1}^{\text {Events }}\left|\mathrm{e}_{\mathrm{i}}-\mathrm{e}_{\mathrm{i}+1}\right|=1
$$


Procedure: Windows between Events

1. Def Windws_Peroids:

2. For All Day Events in Day:

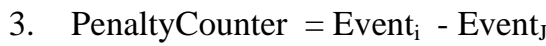

4. IF PenaltyCounter:

5. Layout[Penalty_Slot $]=$ PenaltyCounter $\times$ Scale.Penalty

6. EndIf

7. End_LOOP

\begin{tabular}{|c|c|c|c|c|c|c|}
\hline & & & & & \begin{tabular}{|l} 
Isolated \\
Lectures
\end{tabular} & $\begin{array}{l}\text { Room } \\
\text { Capacity }\end{array}$ \\
\hline Day & Room No & Session - 1 & Session - 2 & \multicolumn{2}{|c|}{ Session - 3} & Session - 4 \\
\hline \multirow{7}{*}{ 초 } & Room 1 & Cur1, S13, T02 & Cur1, s14, T07 & Cur1, & $\mathrm{S} 15, \mathrm{~T} 14$ & \\
\hline & Room 2 & Cur9, S23, T04 & Cur2, S25, T11 & Cur3, & S35, T13 & Cur3, S31, T15 \\
\hline & Room 3 & Cur4, S42, T07 & Cur9, s94, T19 & Cur4, & S44, T11 & Cur4, S41, T06 \\
\hline & Room 4 & Cur5, S53, T05 & Cur5, S55, T12 & Cur5, & S51, T05 & \\
\hline & Room 5 & Cur6, S65, T13 & Cur8, S83, T02 & Cur6, & S61, T15 & \\
\hline & Room 6 & Cur7, s74, T08 & Cur7, s73, T10 & Cur7, & S71, T12 & \\
\hline & Room 7 & Cur8, S81, T14 & Cur6, S62, T14 & Cur10 & S84, T20 & Cur8, S85, T03 \\
\hline
\end{tabular}

Fig 3: Soft Constraints from Scale 1

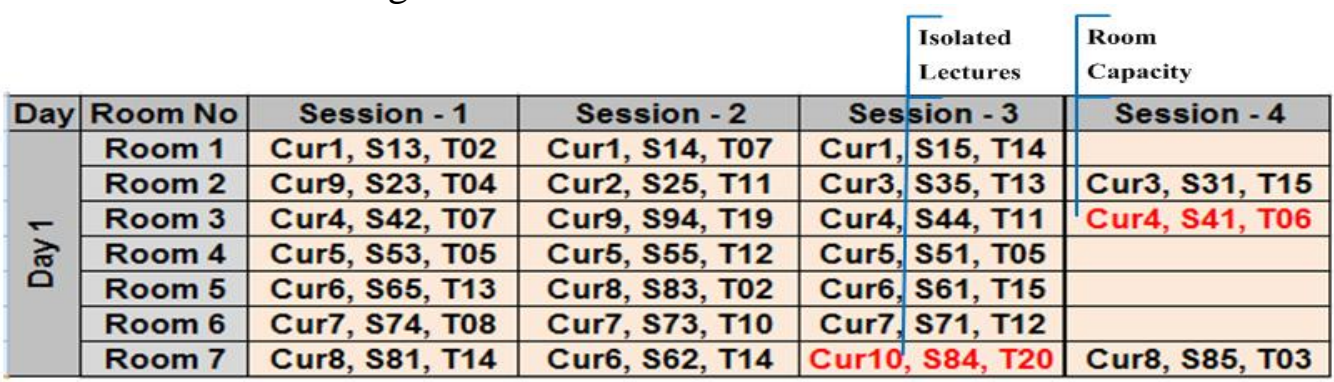

Fig 3: represents the two soft violations from complexity scale 1.

Lecture event in Room 7 of session 3 is only single assignment of that curriculum.

\begin{tabular}{|c|c|c|c|c|c|}
\hline & & & Roon & & \\
\hline Day & Room No & Session-1 & Session - 2 & Session - 3 & Session - 4 \\
\hline & Room 1 & Cur10, S13, T02 & Cur1, S14, T07 & Cur11, S17, T19 & \\
\hline & Room 2 & Cur1, S13, T02 & Cur2, S25, T11 & Cur3, S35, T13 & Cur3, S31, T15 \\
\hline & Room 3 & Cur4, s & Cur9, s94, T19 & Cur1, S15, T14 & Cur4, S41, T06 \\
\hline ส & Room 4 & Cur5, s53, T05 & Cur5, S55, T12 & Cur5, s51, T05 & \\
\hline 0 & Room 5 & Cur6, S65, T13 & Cur8, S83, T02 & Cur6, S61, T15 & \\
\hline & Room 6 & Cur7, s74, T08 & Cur7, s73, T10 & Cur7, s71, T12 & \\
\hline & Room 7 & Cur8, S81, T14 & Cur6, S62, T14 & Cur10, S84, T20 & Cur8, S85, T03 \\
\hline
\end{tabular}

Fig 4 Distinct Soft Constraint of Scale 2 


\begin{tabular}{|c|c|c|c|c|c|}
\hline \multicolumn{5}{|c|}{ Room Stability } & \multirow[b]{2}{*}{ Session - 4} \\
\hline Day & Room No & Session - 1 & Session - 2 & Session - 3 & \\
\hline \multirow{7}{*}{$\frac{5}{\sqrt{0}}$} & Room 1 & Cur10, S13, T02 & Cur1, S14, T07 & Cur11, s17, T19 & \\
\hline & Room 2 & Cur1, S13, T02 & Cur2, S25, T11 & Cur3, S35, T13 & Cur3, S31, T15 \\
\hline & Room 3 & Cur4, S42, T07 & Cur9, s94, T19 & Cur1, S15, T14 & Cur4, s41, T06 \\
\hline & Room 4 & Cur5, S53, T05 & Cur5, s55, T12 & Cur5, S51, T05 & \\
\hline & Room 5 & Cur6, S65, T13 & Cur8, S83, T02 & Cur6, S61, T15 & \\
\hline & Room 6 & Cur7, s74, T08 & Cur7, s73, T10 & Cur7, s71, T12 & \\
\hline & Room 7 & Cur8, S81, T14 & Cur6, S62, T14 & Cur10, s84, T20 & Cur8, S85, T03 \\
\hline
\end{tabular}

Fig. 4: portrays the exclusive properties of complexity scale 2, while the rest of constraints are derived from previous scale.

3.6.2 $\mathrm{SC}_{6}$ (Student MinMax Courses Load): Lecture events must be confined with the limit of (lower and upper bond) sessions assigned to any group of students.

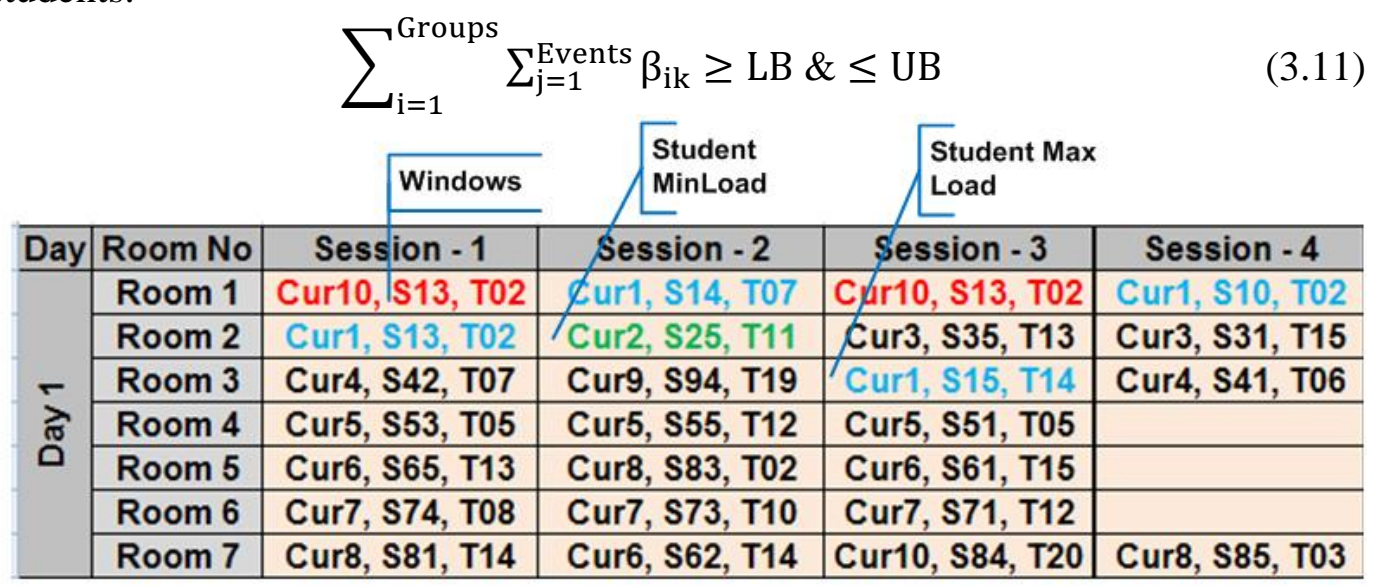

Fig 5: Distinct Soft Constraints from Scale 3

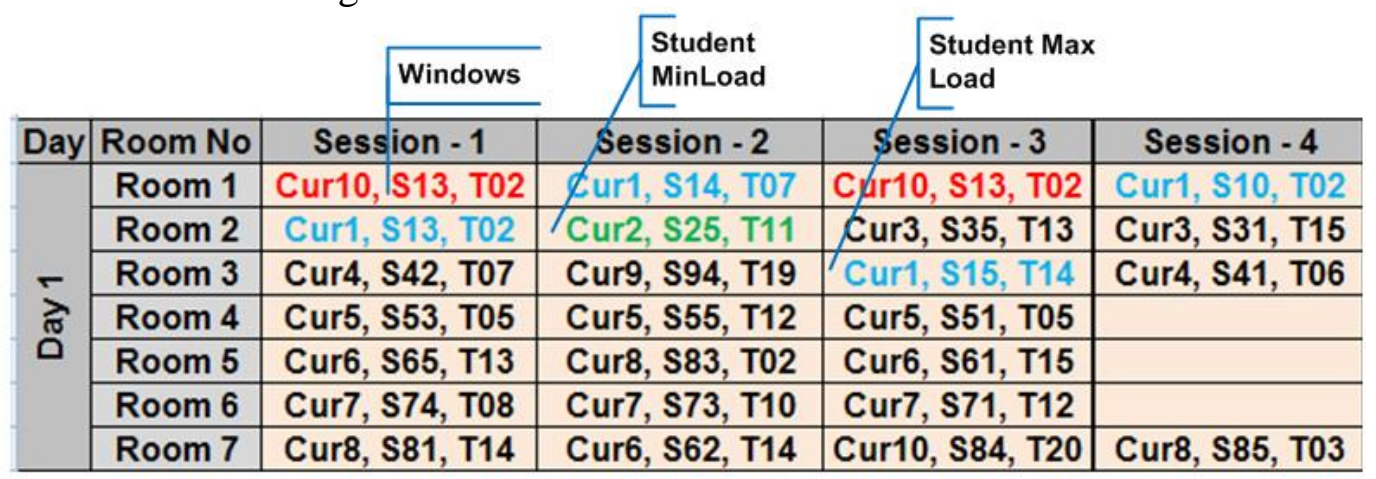

Fig 5: reveals the bespoke constraints of complexity scale 3, other constraints of this scale are shared with previous scales.

Timeslot at location (Room-1, Session-1 and Session-2) clearly exhibits two events of same group in nonconsecutive session; such gap is actually undesirable and counts as soft violation. The cell point (Room-2, Session-2) stands for the 
isolated lecture event. Conversely the lecture events in (Room-2,1,3,1 and Sessions-1,2,3,4 respectively) view the additional number of events assigned in single day for particular curricula which causes the soft violation.

\subsection{Complexity Scale 4}

This scale inherits previous constraints including new one called Double Lecture constraint.

3.7.1 $\mathrm{SC}_{\mathbf{8}}$ (Double Lecture): Sometimes a long sessions or accumulated session is required especially in executive/evening programs or on teacher demand.

$$
\sum_{\mathrm{i}=1}^{\text {Events }} \sum_{\mathrm{j}=1}^{\text {Sessions }}\left|\mathrm{t}_{\mathrm{i}}-\mathrm{t}_{\mathrm{i}+1}\right|=1
$$

\section{Procedure: Double Lecture}

1. Def Windws_Peroids:

2. For All Day Events in Week:

3. SessionGape $_{\mathrm{i}}=\mathrm{Cur}_{\mathrm{i}}$.Event $\mathrm{E}_{\mathrm{i}}-\mathrm{Cur}_{\mathrm{j}}$. Event $\mathrm{j}$

4. IF Not Any $\sum_{1}^{n}$ SessionGape $_{i=0}$

5. Layout[Penalty_Slot $]=$ Scale.Penalty.Double

6. EndIf

7. End_LOOP

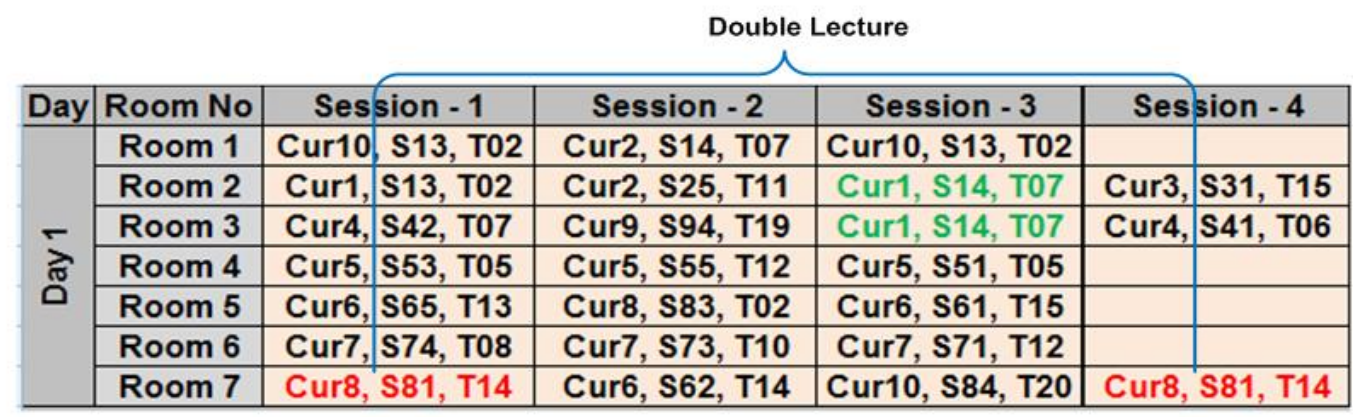

Fig 6: Distinct Sot Scale from Scale 4

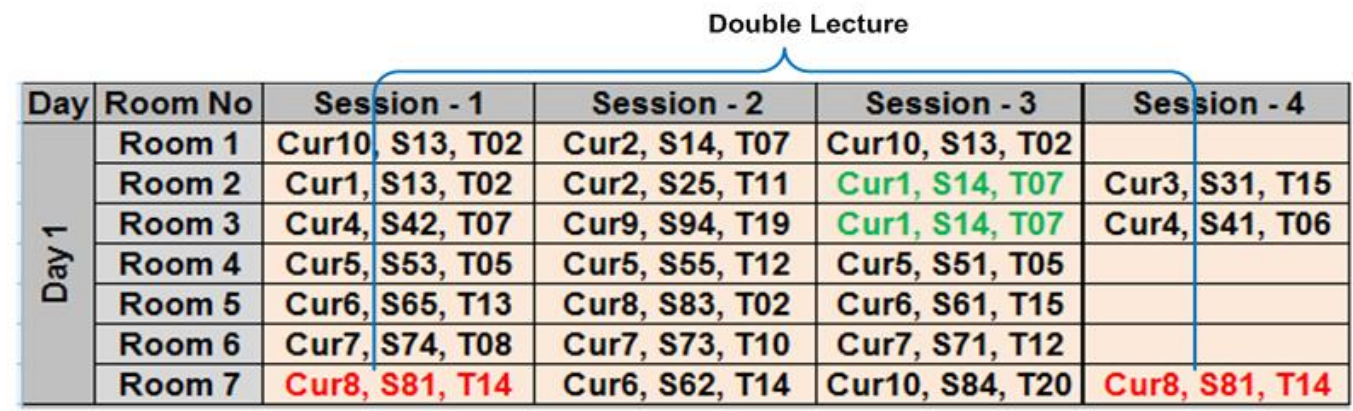

Fig 6: shows the Doubled Lecture constraint violated at (Room-1and 2, Sessions$3)$. 


\subsection{Complexity Scale 5}

The scale addresses the travelling between venue/buildings.

3.8.1. Travel Distance: Conflict violation recorded if events of same group are assigned in different buildings on same day.

$$
\sum_{\mathrm{i}=1}^{\text {Events }} \mathrm{S}_{\mathrm{i}} \cdot \mathrm{b}_{\mathrm{j}}=1 \text { where } \forall \mathrm{j} \in\{1 \cdots \text { Vanue }\}
$$

Procedure: Venue

1. Def Venue_Stability:

2. For All Day Events:

3. IF Sequence_Placments $\left(\right.$ Event $\left._{\mathrm{i}}\right)==$ NOT Same Venue:

4. Layout[Penalty_Slot $]=\sum_{1}^{\mathrm{n}}$ Venue.Violations $\times$ Scale.Penalty

5. EndIf

6. End_LOOP

7. End_LOOP

Travel Distance

\begin{tabular}{|c|c|c|c|c|c|c|}
\hline Day & & Room No & Session - 1 & Session - 2 & Session - 3 & Session - 4 \\
\hline \multirow{7}{*}{ 市 } & \multirow{3}{*}{$\begin{array}{l}\bar{g} \\
\overline{\bar{z}} \\
\overline{\bar{y}}\end{array}$} & Room 1 & Cur10, S13, T02 & Cur2, S14, T07 & Cur10, S13, T02 & \\
\hline & & Room 2 & Cur1, S13, T02 & Cur2, S25, T11 & Cur1, s14, T07 & Cur3, s31, T15 \\
\hline & & Room 3 & Cur4, S42, T07 & Cur8, S81, T14 & Cur1, s14, T07 & Cur4, s41, T06 \\
\hline & \multirow{4}{*}{$\begin{array}{l}\text { N } \\
\text { D्E } \\
\text { 咅 } \\
\overline{\bar{~}}\end{array}$} & Room 4 & Cur5, s53, T05 & Cur5, S55, T12 & Cur5, s51, T05 & \\
\hline & & Room 5 & Cur6, S65, T13 & Cur8, S83, T02 & Cur6, S61, T15 & \\
\hline & & Room 6 & Cur7, s74, T08 & Cur7, s73, T10 & Cur7, s71, T12 & \\
\hline & & Room 7 & Cur8, S81, T14 & Cur6, S62, T14 & Cur10, S84, T20 & \\
\hline
\end{tabular}

Fig 7 Soft Constraint from Scale 6

Fig 7 portrays the timetabling divided into two building so consequently on some it causes the violations.

\subsection{Complexity Scale 6}

The scale originates a new constraint Teaching Work Load along with rest of others.

3.9.1 Teaching Work Load: If teaching work assignments go beyond the upper bond it may be raised with identical number of violations.

$$
\sum_{\mathrm{i}=1}^{\text {Faculty }} \mathrm{S}_{\mathrm{i}} \cdot \mathrm{b}_{\mathrm{j}} \leq \mathrm{LB} \text { where } \forall \mathrm{j} \in\{1 \cdots \text { TeachingLoad }\}
$$


Procedure: Teacher Max Load

1. Def TeacherMaxLoad:

2. For Scan DayEvents:

3. $\quad$ Range $=$ Counts $\left(\sum_{1}^{\mathrm{n}}\right.$ Teacher. Events $)$

4. IF Range > TeacherLoad.UB:

5. Layout $[$ Penalty_Slot $]=$ Scale.Penalty $\times$ Range - TeacherLoad.UB

6. EndIf

7. End_LOOP

\begin{tabular}{|c|c|c|c|c|c|c|c|}
\hline \multicolumn{8}{|c|}{$\begin{array}{l}\text { Teaching } \\
\text { Load }\end{array}$} \\
\hline Day & & Room No & Session - 1 & Ses & sion - 2 & Session - 3 & Session - 4 \\
\hline \multirow{7}{*}{ 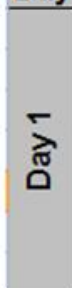 } & \multirow{3}{*}{$\begin{array}{l}\bar{Z} \\
\overline{\bar{z}} \\
\overline{\bar{c}}\end{array}$} & Room 1 & Cur10, S13, T02 & Cur2, & S14, T07 & Cur10, s13, T02 & \\
\hline & & Room 2 & Cur1, S13, T02 & Cur2, & $\mathrm{S} 25, \mathrm{~T} 11$ & Cur1, s14, T07 & Cur3, S31, T15 \\
\hline & & Room 3 & Cur4, S42, T07 & Cur8, & $\mathrm{S} 81, \mathrm{~T} 14$ & Cur1, S14, T07 & Cur4, S41, T06 \\
\hline & \multirow{4}{*}{ 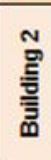 } & Room 4 & Cur5, S53, T05 & Cur5, & S55, T12 & Cur5, S51, T05 & Cur5, s51, T05 \\
\hline & & Room 5 & Cur6, S65, T13 & Cur8, & S83, T02 & Cur6, S61, T15 & \\
\hline & & Room 6 & Cur7, s74, T08 & Gurt, & $\mathrm{S73}, \mathrm{T10}$ & Cur7, s71, T12 & \\
\hline & & Room 7 & Cur8, s81, T14 & Cur6, & S62, T14 & Cur6, S60, T14 & \\
\hline
\end{tabular}

Fig 1 Distinguished Soft Constraints

\begin{tabular}{|c|c|c|c|c|c|c|c|}
\hline \multicolumn{8}{|c|}{$\begin{array}{l}\text { Teaching } \\
\text { Load }\end{array}$} \\
\hline Day & & Room No & Session - 1 & Ses & sion - 2 & Session - 3 & Session - 4 \\
\hline \multirow{7}{*}{ 离 } & \multirow{3}{*}{ 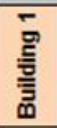 } & Room 1 & Cur10, S13, T02 & Cur2, & S14, T07 & Cur10, S13, T02 & \\
\hline & & Room 2 & Cur1, S13, T02 & Cur2, & $\mathrm{S} 25, \mathrm{~T} 11$ & Cur1, s14, T07 & Cur3, S31, T15 \\
\hline & & Room 3 & Cur4, S42, T07 & Cur8, & $\mathrm{S} 81, \mathrm{~T} 14$ & Cur1, S14, T07 & Cur4, S41, T06 \\
\hline & \multirow{4}{*}{ 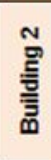 } & Room 4 & Cur5, S53, T05 & Cur5, & S55, T12 & Cur5, s51, T05 & Cur5, s51, T05 \\
\hline & & Room 5 & Cur6, S65, T13 & Cur8, & $\mathrm{S} 83, \mathrm{~T} 02$ & Cur6, S61, T15 & \\
\hline & & Room 6 & Cur7, s74, T08 & Gur7, & $\mathrm{S} 73, \mathrm{~T} 10$ & Cur7, s71, T12 & \\
\hline & & Room 7 & Cur8, S81, T14 & Cur6, & S62, T14 & Cur6, S60, T14 & \\
\hline
\end{tabular}

Fig 1 demonstrates the three consecutive teaching events in such a case where upper limit is declared as two lectures per day.

\section{Results}

The experiential outcome of evaluation logic/function validates the accuracy and efficiency. The computational approach is investigated over a wide range of benchmark datasets. Table 2 portrays the results of the ten benchmark instances (classified over six separate complexity scales). All the problem instances differ from each other on the basis of various parameters counting problem depth, venue saturation and resource limitations etc.

Complexity Scale -1 encloses four permanent hard constraints $\left(\mathrm{HC}_{1}, \mathrm{HC}_{2}, \mathrm{HC}_{3}\right.$ and $\left.\mathrm{HC}_{4}\right)$ and also three soft constraints $\left(\mathrm{SC}_{1}, \mathrm{SC}_{2}\right.$ and $\left.\mathrm{SC}_{3}\right)$. The evaluation 
function (coded in python language) extract out the violations against all types of constraints. In particular, the constraint $\mathrm{HC}_{4}$ is removed due to scheduling layout design and placement of events.

The $\mathrm{HC}_{2}$ has comparatively bigger chance of occurrence replications as enormous numbers of courses are shared by various curriculums simultaneously. Usually, the $\mathrm{HC}_{5}$ violation happened when shared or visiting faculty is involved. It is mandatory in all the scales to eliminate hard constraints first. A single hard constraint violation even stands solution misfit/unfeasible. In complexity scale-1 the soft constrain $\left(\mathrm{SC}_{1}\right)$ identifies the Room Capacity. It was elaborated that each added student more than the available seats in class room increases violation counter. $\mathrm{SC}_{2}$ and $\mathrm{SC}_{3}$ are also showing their presence in the datasets. Total penalty costs of each complexity scale mounts with respect to dataset, due to increasing number of events, saturation and escalating complexity.

Scale-4 in Table 2 demonstrates the Double Lectures $\left(\mathrm{SC}_{8}\right)$ constraints; the provision sometimes is required by visiting faculty. Travel distance $\left(\mathrm{SC}_{7}\right)$ is another important constraint which is shown in higher level of Scale number 5. Scale-6 is representing work load constraint named Teaching Load $\left(\mathrm{SC}_{9}\right)$. The removal of constraint violation provides comfort to teachers to produce quality work.

\section{Conclusions}

The Research work has addressed important issues including the evaluation function and penalty schema. The vast manmade of relevant field and computational experience is incorporated for designing the evaluation function (set of heuristics). Promising outcome is acquired by implementation on the various benchmark datasets which evidently endorse the accuracy in research direction.

\section{References}

[1] Aftab Ahmed, Bukhari, A. H. S. \&Ismaili, I. A. (2011a). A Compartive Study On Three Hyper-Heuristic Approaches For Solving Benchmark Scheduling Problems Sindh University Research Journal (Science Series) 43(2): 161-168.

[2] Aftab Ahmed \&Li, Z. (2010).A Biphasic Approach for University Timetabling Problem. In The 2nd International Conference on Computer Engineering and Technology (ICCET 2010), Vol. 1, 192-197 Chengdu, Sichuan, China.

[3] Aftab Ahmed, Zhoujun Li \&Bukhari, A. H. S. (2011b).Triphasic solving approach for scheduling problem. In Seventh International Conference on Natural Computation (ICNC), 20112358 - 2363 Shanghai, China IEEE.

[4] Alex Bonutti, Fabio De Cesco, Luca Di Gaspero \&Schaerf, A. (2010). Benchmarking curriculum-based course timetabling: formulations, data formats, instances, validation, visualization, and results Annals of Operations Research 179.

[5] G. M. White \&J. Zhang (1998).Generating Complete University Timetables by Combining Tabu Search with Constraint Logic. In The Practice and Theory of Automated Timetableing PATAT 1997, 187-198: Springer-Verlag. 
[6] Ghaemi, S., Vakili, M. T. \&Aghagolzadeh, A. (2007).Using a genetic algorithm optimizer tool to solve University timetable scheduling problem. In ISSPA 07: IEEE.

[7] M. Adriaen, P. De Causmaecker \&Berghe, G. V. (2003).Decentralised course timetabling in a large hierarchical organisation. In Proceedings of 1 st Multidisciplinary International Conference on Scheduling: Practice and Theory, MISTA 2003, 60-66 Nottingham.

[8] M.W. Carter \&Laporte, G. (1998).Recent developments in practical course timetabling. In Second International Conference on the Practice and Theory of Automated Timetabling (PATAT II), 3-19 (Eds E.K. Burke and M. Carter). Canada.

[9] W. Legierski (2002).Constraint-based reasoning for timetabling. In AI-METH 2002Gliwice, Poland. 
Table 2 Results of Evaluating Constraints of 10 datasets

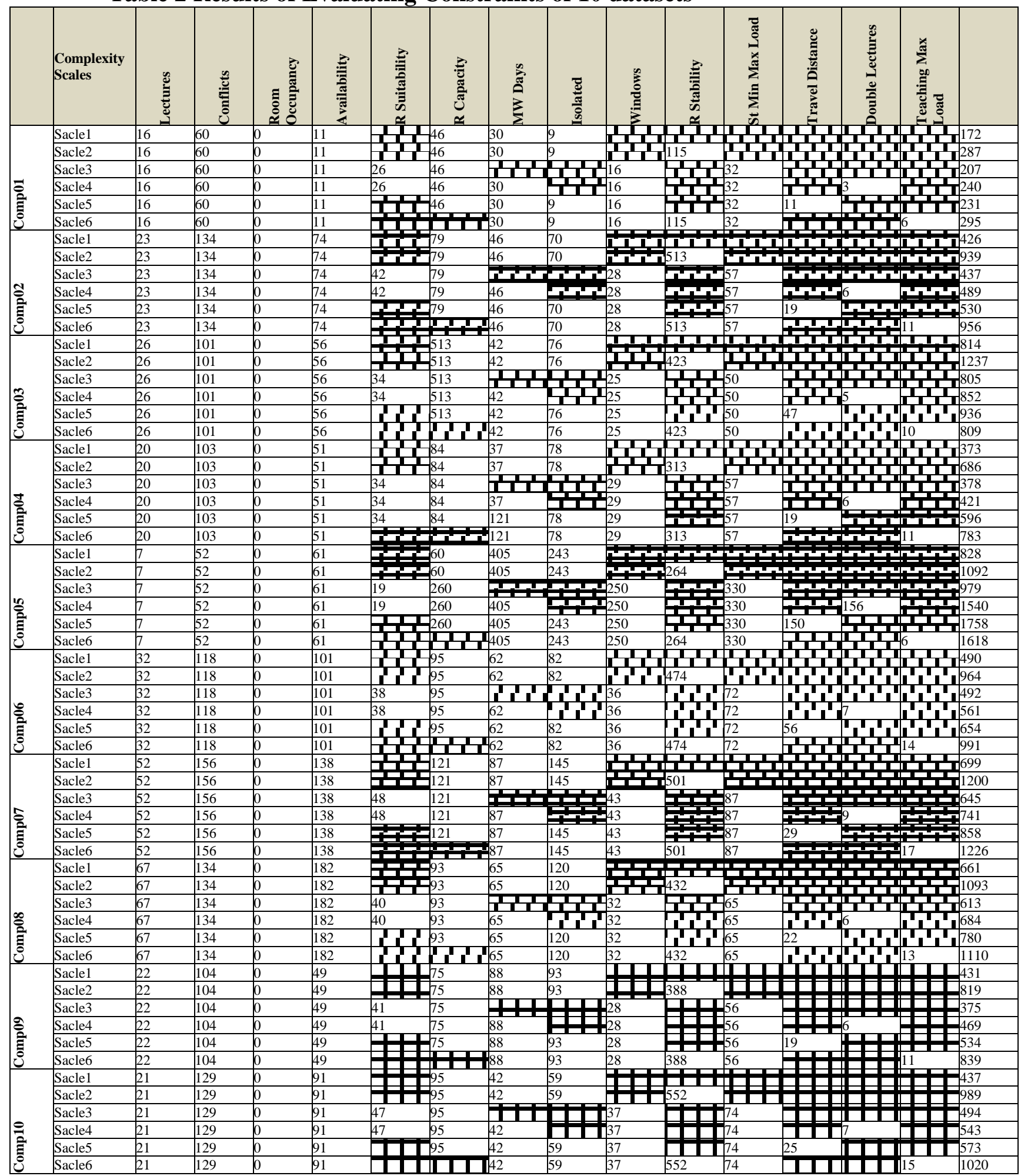

